\title{
Pressure Change of the Phase Diagram of the Binary Mixture of Low Molecular Weight Polyethylenes
}

\author{
Chitoshi NaKafuku \\ Faculty of Education, Kochi University, Kochi 780, Japan
}

(Received March 16, 1995)

\begin{abstract}
Effects of pressure on the phase behaviors of the three polyethylene(PE)-polyethylene(PE) binary mixtures with different molecular weights $(M W=10000,2000$, and 1000$)$ were studied. High pressure differential thermal analysis (DTA) was performed at pressures up to $500 \mathrm{MPa}$. For the three binary mixtures, the melting temperature $T_{\mathrm{m}}$ of the higher molecular weight PE decreased with decreasing the weight fraction of the high molecular weight PE, while $T_{\mathrm{m}}$ of the PEs with lower $M W$ of 1000 and 2000 did not change with the weight fraction. At elevated pressure, the phase diagram resulted in an increase of the difference between $T_{\mathrm{m}}$ of the higher molecular weight PE and that of the lower molecular weight PE. The phase diagram of melting of PE 2000 and PE 1000 binary mixture was eutectic type at $0.1 \mathrm{MPa}$. The eutectic point did not change with pressure. Effects of mixing PE 1000 and PE 2000 on the appearance of the high pressure phase and the formation of the extended chain crystal of PE 10000 was studied as a function of the weight fraction. The molecular mechanism of the melting and crystallization process of PE in the binary mixture under high pressure was considered.

KEY WORDS Polyethylene / Low Molecular Weight / Binary Mixture / High Pressure / Melting / Crystallization / Differential Thermal Analysis / Phase Diagram / Extended Chain Crystal /
\end{abstract}

The phase diagram of melting of $n$-alkane $/ n$ alkane and poly(ethylene)(PE)/ $n$-alkane binary mixture at atmospheric pressure has been studied by many authors. ${ }^{1-9}$ The $n$-alkane $/ n$ alkane binary mixture solidifies in solid solution or two solid phases depending on the molecular weight difference of the constituents or cooling rates from the molten state. In the $\mathrm{PE} / n$-alkane mixture, melting point $\left(T_{\mathrm{m}}\right)$ of $\mathrm{PE}$ decreased with decreasing the content in the mixture.

Recently, we studied the pressure change of the phase diagram of the binary mixture of $n$-triacontane $(\mathrm{C} 30)$ and $n$-docosane(C22) showing eutectic phase diagram and that of $\mathrm{C} 30$ and $n$-hexacosane (C26) showing solid solution type phase diagram up to $500 \mathrm{MPa} .{ }^{10}$ The phase diagram for both binary mixtures changed with pressure.

Effect of mixing tetracontane (C40) on the melting and crystallization of PE of normal molecular weight (NMW-PE) of the molecular weight 67000 has been studied at elevated pressures up to $500 \mathrm{MPa} .{ }^{11} T_{\mathrm{m}}$ of NMW-PE decreased with increasing $\mathrm{C} 40$ content in the mixture at $0.1 \mathrm{MPa}$ and the temperature difference between the $T_{\mathrm{m}}$ of NMW-PE and C40 increased with pressure. The high pressure phase of PE is also affected by mixing C40. The area of the high pressure phase of NMW$P E$ decreased with increasing the weight fraction of $\mathrm{C} 40$. The high pressure phase disappeared at the weight fraction of PE ( $W(\mathrm{PE}))$ $=0.4$.

It is interesting to study the effect of pressure on the phase diagram of the binary mixture of low molecular weight PEs that cover the molecular weight range between $n$-alkane and NMW-PE.

In this paper, melting and crystallization 
behaviors of the three binary mixtures of $\mathrm{PE}$ of the molecular weight 10000(PE10T), 2000 (PE2T), and 1000(PE1T) were studied under high pressure. The three phase diagrams on the combinations of the above three PE samples were obtained at $0.1 \mathrm{MPa}$ and high pressure. The behavior of these phase diagrams under high pressure was compared with that of the phase diagram of NMW-PE/C40 mixture and $n$-alkane/n-alkane mixture.

Under high pressure above $350 \mathrm{MPa}$, phase transition from the orthorhombic to the hexagonal phase is observed in NMW-PE. Furthermore, if PE is crystallized from the melt under high pressure above $350 \mathrm{MPa}$, so called extended chain crystal (ECC) is formed. Effect of mixing PE1T and PE2T on the phase transition and ECC formation of PE10T under high pressure is studied.

\section{EXPERIMENTAL}

A powder sample of PE10T (Hizex SPX001, $\left.M W=10000, \quad M_{w} / M_{n}=7.42\right)$ supplied by Mitsui Petrochemical Co. was used. PE1T $\left(M W=1000, M_{w} / M_{n}=1.10\right)$ and $\operatorname{PE} 2 \mathrm{~T}(M W=$ 2000, $\left.M_{w} / M_{n}=1.10\right)$ purchased from Polymer Laboratories, Inc. were used. In order to make the binary mixture, powder samples were mixed at a desired weight fraction and were put into a glass tube of $1.8 \mathrm{~mm}$ in inside diameter. The sample was melted at $160^{\circ} \mathrm{C}$ and then cooled to room temperature at a rate of about $1.2 \mathrm{~K} \mathrm{~min}^{-1}$.

High pressure DTA was performed using an apparatus described elsewhere. ${ }^{11}$ The rod-like sample $1.8 \mathrm{~mm}$ in diameter was cut to $1.8 \mathrm{~mm}$ in length and wrapped by aluminum foil $(0.01 \mathrm{~mm}$ in thickness) and attached to a thermocouple junction of the DTA apparatus. DTA curve of melting and crystallization was obtained by the following way. At a desired pressure, the sample was heated to a temperature above $T_{\mathrm{m}}$ at the rate of $6 \mathrm{~K} \mathrm{~min}^{-1}$. High pressure crystallization was performed by cooling the sample from above $T_{\mathrm{m}}$ at the pressure. The cooling rate was $4-5 \mathrm{~K} \mathrm{~min}^{-1}$.

DSC measurements were performed by using a Rigaku low temperature thermal analyzer (DSC 8230D) at a heating rate of $5 \mathrm{~K} \mathrm{~min}^{-1}$.

Wide angle X-ray diffraction (WAXD) pattern of the mixture was obtained in the heating process on the rod-like sample covered by aluminum foil. $\mathrm{Cu}-K_{\alpha}$ radiation was used as an incident X-ray beam. The sample was set in the heating device on the goniometer and the temperature of the sample was detected by an alumel-chromel thermocouple attached to the foil-covered sample. The heating rate was about $6 \mathrm{~K} \mathrm{~min}^{-1}$.

\section{RESULTS AND DISCUSSION}

Pressure Dependence of the Phase Diagram of the Three Binary Mixtures

Figure 1 shows the DSC melting curves of the binary mixture of PE10T and PE1T with different weight fractions. The high temper-

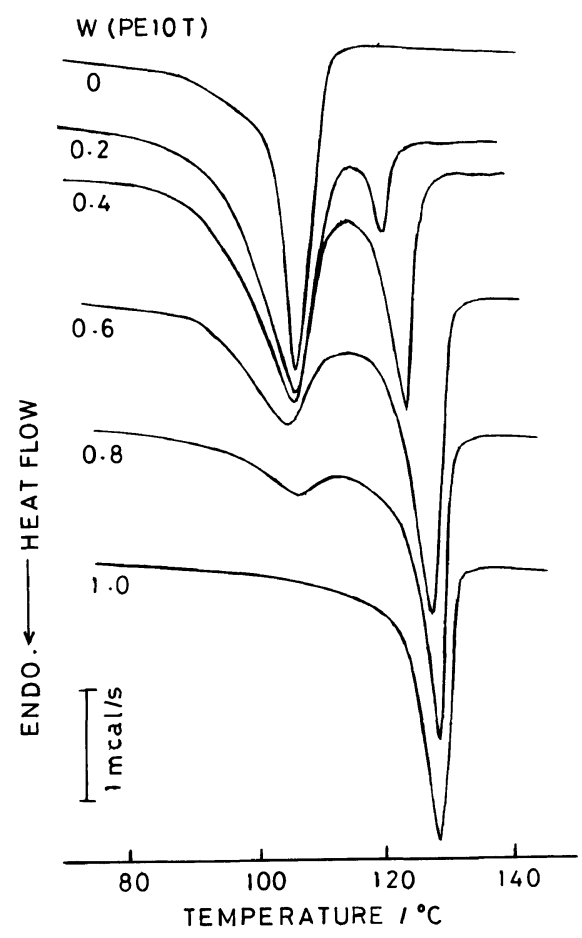

Figure 1. DSC curve of melting of the binary mixture of PE10T and PE1T with different weight fractions. 


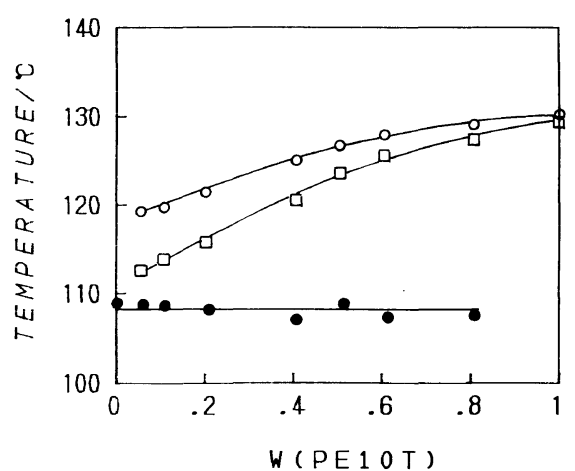

Figure 2. Phase diagram of melting of PE10T/PE1T binary mixture. $\mathrm{O}, \mathrm{PE} 10 \mathrm{~T}$ in the sample cooled slowly $\left(4 \mathrm{~K} \mathrm{~min}^{-1}\right) ; \square$, PE10T in the sample quenched to ice water; $\mathrm{O}, \mathrm{PE} 1 \mathrm{~T}$ in the sample cooled slowly.

ature endothermic peak is due to the melting of PE10T and the low temperature peak at about $105^{\circ} \mathrm{C}$ is due to the melting of PE1T. The peak intensity of melting of PE10T decreased with decreasing PE10T content in the mixture. The high temperature peak shifted to low temperature with decreasing PE10T content and the trend is radical below $W(\mathrm{PE} 10 \mathrm{~T})$ $=0.6$, while the low temperature peak did not shift with the content. The phase diagram of PE10T/PE1T binary mixture at atmospheric pressure is obtained by plotting the melting peak temperature against weight fraction of PE10T $(W(\mathrm{PE} 10 \mathrm{~T}))$ as shown in Figure 2. $T_{\mathrm{m}}$ of PE10T clearly decreased with decreasing PE10T content. In the quenched sample, the decreasing rate is larger than that of the samples slowly cooled from the melt indicating the formation of small crystals of PE10T in quenching. Solid solution was not formed in this binary mixture.

The temperature change of the WAXD pattern of pure PE10T and the binary mixture was studied in the heating process. Figure 3(a) shows the WAXD pattern of pure PE10T at elevated temperature. The intensity of the (110) and (200) diffraction peak did not change at temperatures up to $90^{\circ} \mathrm{C}$ but the intensity decreased at $120^{\circ} \mathrm{C}$ just below the melting temperature. At $130^{\circ} \mathrm{C}$, the diffraction peak (a)

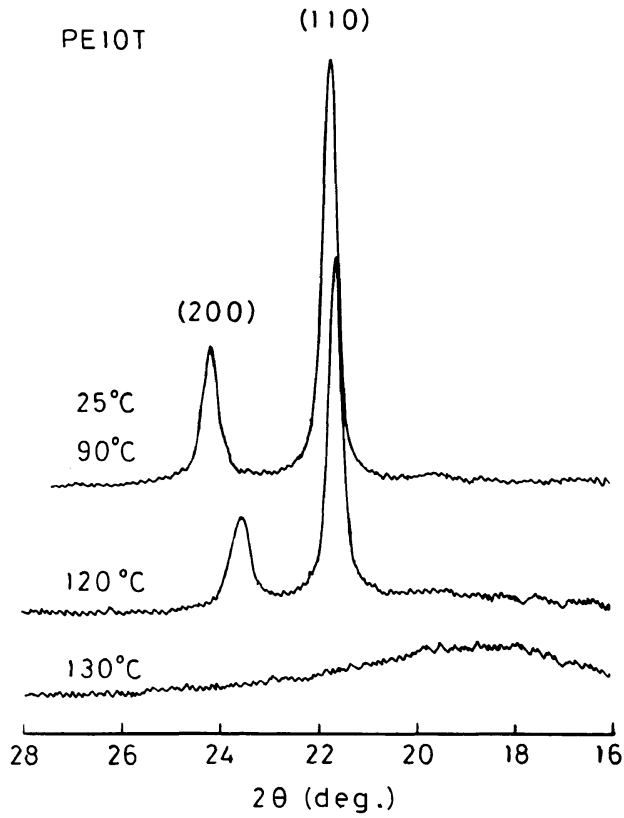

(b)

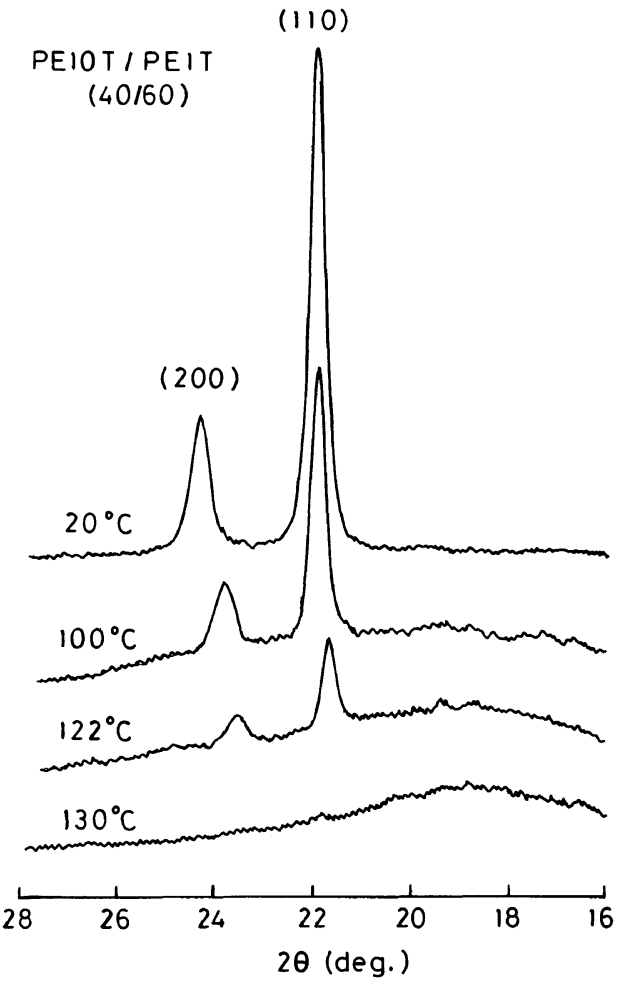

Figure 3. Temperature change of the X-ray diffraction pattern of (a) pure PE10T and (b) PE10T/PE1T binary mixture of $W(\mathrm{PE} 10 \mathrm{~T})=0.4$ 


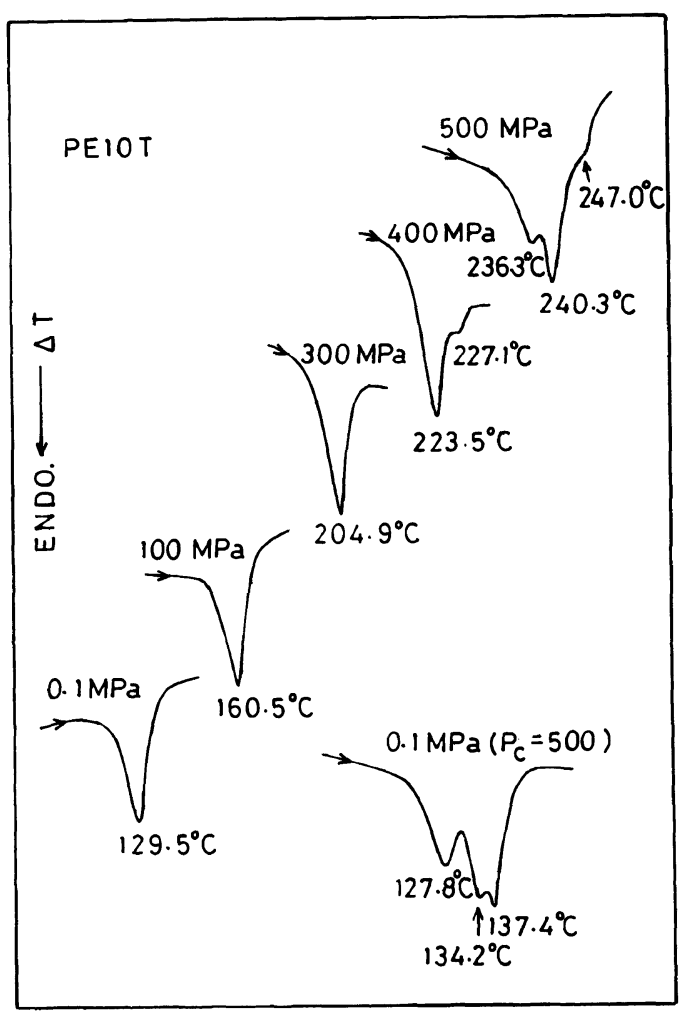

Figure 4. Pressure change of the DTA curve of melting of pure PE10T. The DTA curve of melting of the sample crystallized at $500 \mathrm{MPa}$ is shown at the bottom.

disappeared. The DSC curve of pure PE10T shows the starting of melting at $120^{\circ} \mathrm{C}$, so that the decrease of the diffraction intensity coincides with the thermal behavior. As shown in Figure 3(b), the intensity of the diffraction peak of PE10T/PE1T binary mixture of $W(\mathrm{PE} 10 \mathrm{~T})=0.4$ decreased at $100^{\circ} \mathrm{C}$ corresponding to the low temperature endothermic peak of melting in the DSC curve. At $122^{\circ} \mathrm{C}$, the diffraction intensities become very small.

Figure 4 shows the pressure change of the DTA curve of melting of pure PE10T. Single endothermic peak of melting is observed at pressures up to $350 \mathrm{MPa}$. The peak becomes multiple peaks above $400 \mathrm{MPa}$ due to the formation of high pressure phase. The three endothermic peaks observed at $500 \mathrm{MPa}$ are already ascertained. The highest peak at

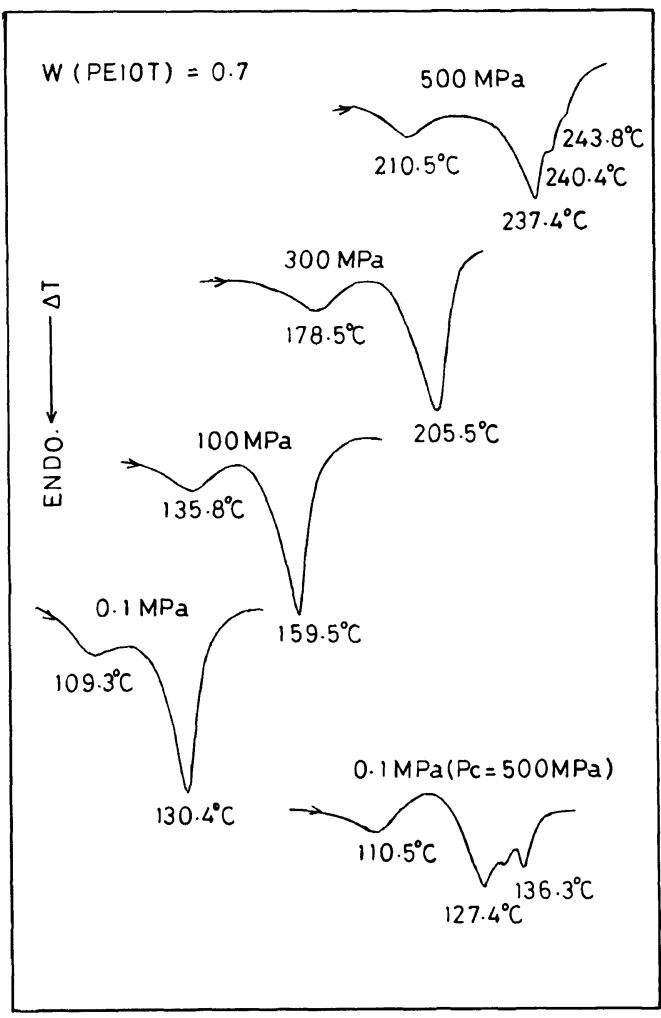

Figure 5. Pressure change of the DTA curve of melting of the binary mixture of $W(\mathrm{PE} 10 \mathrm{~T})=0.7$. The DTA curve of melting of the sample crystallized at $500 \mathrm{MPa}$ is shown at the bottom.

$247.0^{\circ} \mathrm{C}$ is due to the melting of the hexagonal phase and the peak at $240.3^{\circ} \mathrm{C}$ is due to the phase transition. It is recognized that ECC is formed in PE10T by high pressure crystallization taking into account that the high temperature peak $\left(137.4^{\circ} \mathrm{C}\right)$ is observed in the DTA curve of melting at $0.1 \mathrm{MPa}$ on the sample crystallized at $500 \mathrm{MPa}$ as shown in the bottom of Figure 4.

Figure 5 shows the pressure change of the DTA melting curves of the PE10T/PE1T binary mixture at $W(\mathrm{PE} 10 \mathrm{~T})=0.7$. Two endothermic peaks due to the melting of PE1T (low temperature peak) and PE10T (high temperature peak) appears up to $400 \mathrm{MPa}$. At $500 \mathrm{MPa}$, the melting peak of PE10T is composed of three peaks due to the melting of the 
folded chain crystal (FCC) at $237.4^{\circ} \mathrm{C}$, the phase transition at $240.4^{\circ} \mathrm{C}$ and the melting of the high pressure phase at $243.8^{\circ} \mathrm{C}$. The DTA curve of melting and crystallization under high pressures up to $500 \mathrm{MPa}$ was obtained for all the binary mixtures with different weight fractions. The peak due to the phase transition becomes small with decreasing PE10T content in the mixture. In this mixture of $W(\mathrm{PE} 10 \mathrm{~T})=$ 0.7 , ECC of PE10T is also formed by cooling from the melt at $500 \mathrm{MPa}$ as observed in the DTA curve of melting of the sample crystallized at $500 \mathrm{MPa}$ as shown at the bottom in Figure 5. The highest temperature peak at $136.3^{\circ} \mathrm{C}$ is about $8^{\circ} \mathrm{C}$ higher than $T_{m}$ of FCC of PE10T $\left(127.4^{\circ} \mathrm{C}\right)$. In pure PE1T, the peak intensity and peak shape did not change with pressure up to $500 \mathrm{MPa}$. Therefore, high pressure phase transition did not occur in PE of the molecular weight 1000 .

Figure 6 shows the pressure dependence of $T_{\mathrm{m}}$ of pure PE10T and PE10T and PE1T in the binary mixture of $W(\mathrm{PE} 10 \mathrm{~T})=0.7$. Above $400 \mathrm{MPa}$, the low temperature peak for the melting of PE10T is plotted against pressure in the figure. The curve fits the quadratic equation, $T_{\mathrm{m}}=A+B P-C P^{2}$ and the coefficients $A, B$, and $C$ were determined by the least squares method using the data up to $500 \mathrm{MPa}$. The melting curve ( $T_{\mathrm{m}}$ versus pressure curve) was obtained for the mixtures with different weight fractions. The values of $A, B$, and $C$ for pure PE10T and PE1T and the values for PE10T and PE1T in the binary mixture are listed in Table I. The value of $A$ that means $T_{\mathrm{m}}$ of PE10T at $0.1 \mathrm{MPa}$ decreased with decreasing PE10T content but that of PE1T is almost constant with the change of the weight fraction. The value of $B$ that means the pressure dependence of $T_{\mathrm{m}}\left(\mathrm{d} T_{\mathrm{m}} / \mathrm{d} P\right)$ at $0.1 \mathrm{MPa}$ does not change with the weight fraction of PE10T and PE1T. The value of $B$ for PE10T is about $0.30 \mathrm{~K} \mathrm{MPa}^{-1}$ which is the same as that of NMW-PE already reported. ${ }^{11}$ The value of about $0.28 \mathrm{~K} \mathrm{MP}^{-1}$ for $B$ of PE1T is between those of PE10T and $n$-alkanes (about 0.25

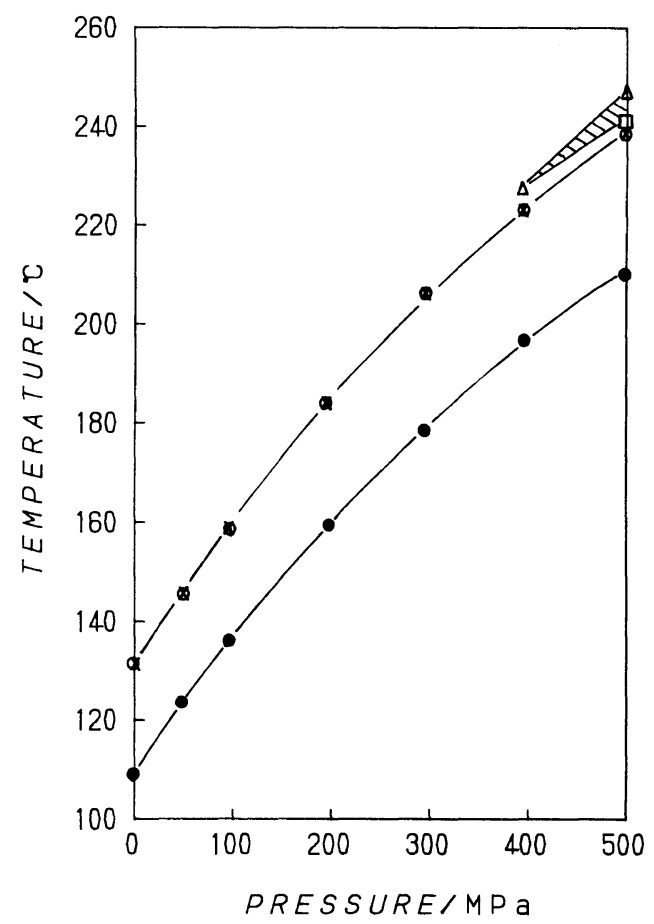

Figure 6. Pressure dependence of $T_{\mathrm{m}}$ of pure PE10T, PE10T, and PE1T in the mixture with $W(\mathrm{PE} 10 \mathrm{~T})=0.7$. The hatched area shows the high pressure phase of PE10T. $\bigcirc$, pure PE10T; $\times$, PE10T in the mixture; $\boldsymbol{O}$, PE1T in the mixture ; $\triangle$, melting of the high pressure phase; $\square$, phase transition.

$\left.\mathrm{K} \mathrm{MPa}^{-1}\right)$. In the mixture of $W(\mathrm{PE} 10 \mathrm{~T})=0.9$ and 0.8 , the values of PE1T could not be determined because the melting peak did not appear. The hatched area shows the high pressure phase of PE10T.

The phase diagram of the binary mixture at a desired pressure can be obtained by plotting the values of $T_{\mathrm{m}}$ calculated from the quadratic equation using the values of Table I. Figures 7(a) and (b) show the phase diagram of the binary mixture of PE10T and PE1T at 0.1 and $500 \mathrm{MPa}$, respectively. $T_{\mathrm{m}}$ depression rate of PE10T decreased at elevated pressure but $T_{\mathrm{m}}$ of PE1T did not change with the weight fraction up to $500 \mathrm{MPa}$. The temperature difference between $T_{\mathrm{m}}$ of PE10T and PE1T increased with pressure. This means that the solvent effect of PE1T decreases with pressure. It is considered that the molecular interaction 


\section{NAKAFUKU}

Table I. Values of $A, B$, and $C$ in the quadratic equation, $T_{\mathrm{m}}=A+B P-C P^{2}$ for the melting temperature of PE10T and PE1T in the mixture for different weight fraction $\left(W_{\mathrm{PE} 10 \mathrm{~T}}=1.0-0.0\right)$

in run 1 of DTA up to $500 \mathrm{MPa}$

$\left(\begin{array}{c}\text { For } W_{\mathrm{PE} 10 \mathrm{~T}}=0.7-1.0, \text { the values are determined by the data of } T_{\mathrm{m}} \text { up to } 350 \mathrm{MPa} \text { and } \\ \text { that of low temperature peak above } 400 \mathrm{MPa} .\end{array}\right)$

\begin{tabular}{|c|c|c|c|c|c|c|}
\hline \multirow{3}{*}{$W_{\mathrm{PE} 10 \mathrm{~T}}$} & \multicolumn{3}{|c|}{ PE10T } & \multicolumn{3}{|c|}{ PE1T } \\
\hline & $A$ & $B$ & $C$ & $A$ & $B$ & $C$ \\
\hline & ${ }^{\circ} \mathrm{C}$ & $\mathrm{K} \mathrm{MPa}^{-1}$ & $\mathrm{KMPa}^{-2}$ & ${ }^{\circ} \mathrm{C}$ & $\mathrm{K} \mathrm{MPa}^{-1}$ & $\mathrm{KMPa}^{-2}$ \\
\hline 1.0 & 131.0 & 0.302 & $1.729 \times 10^{-4}$ & & & \\
\hline 0.9 & 130.8 & 0.303 & $1.770 \times 10^{-4}$ & & & \\
\hline 0.8 & 131.7 & 0.293 & $1.549 \times 10^{-4}$ & & & \\
\hline 0.7 & 131.0 & 0.296 & $1.577 \times 10^{-4}$ & 109.7 & 0.279 & $1.568 \times 10^{-4}$ \\
\hline 0.6 & 127.1 & 0.303 & $1.803 \times 10^{-4}$ & 108.9 & 0.280 & $1.602 \times 10^{-4}$ \\
\hline 0.5 & 126.4 & 0.306 & $1.827 \times 10^{-4}$ & 109.2 & 0.282 & $1.623 \times 10^{-4}$ \\
\hline 0.4 & 125.2 & 0.301 & $1.729 \times 10^{-4}$ & 108.9 & 0.274 & $1.447 \times 10^{-4}$ \\
\hline 0.3 & 125.3 & 0.305 & $1.845 \times 10^{-4}$ & 110.2 & 0.279 & $1.659 \times 10^{-4}$ \\
\hline 0.2 & 121.6 & 0.306 & $1.775 \times 10^{-4}$ & 109.5 & 0.284 & $1.669 \times 10^{-4}$ \\
\hline 0.1 & 121.4 & 0.293 & $1.637 \times 10^{-4}$ & 111.0 & 0.276 & $1.523 \times 10^{-4}$ \\
\hline 0.0 & & & & 111.6 & 0.279 & $1.645 \times 10^{-4}$ \\
\hline
\end{tabular}

(a)

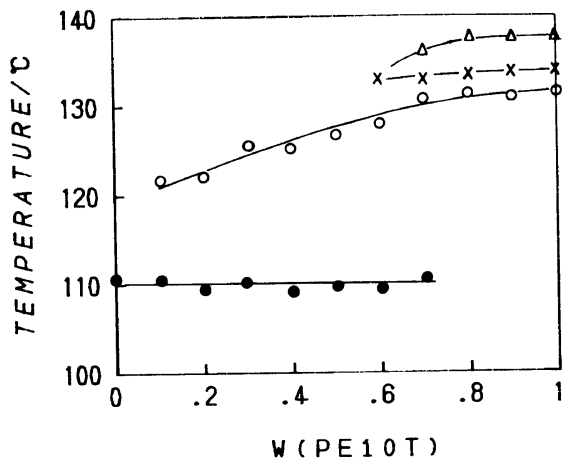

(b)

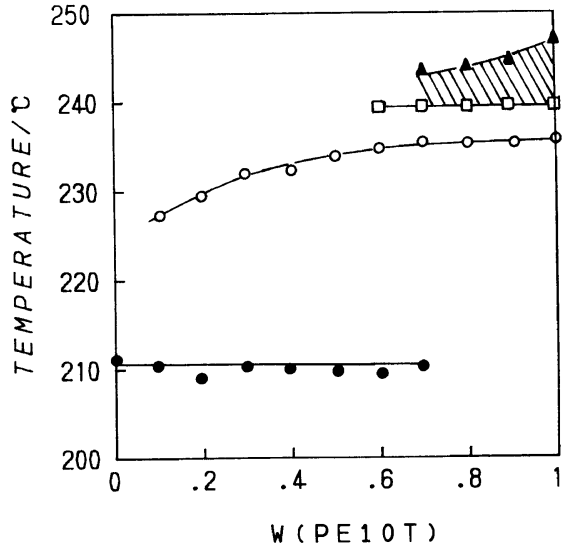

Figure 7. (a): Phase diagram of melting of the binary mixture of PE10T and PE1T at $0.1 \mathrm{MPa}$. $\mathrm{O}, \mathrm{PE} 10 \mathrm{~T}$ crystallized at $0.1 \mathrm{MPa} ; \mathrm{PE} 1 \mathrm{~T} ; \times, \triangle, \mathrm{PE} 10 \mathrm{~T}$ crystallized at $500 \mathrm{MPa}$.

(b): Phase diagram of melting of the binary mixture of E10T and PE1T at $500 \mathrm{MPa}$. $\bigcirc$, PE10T crystallized at $0.1 \mathrm{MPa}$; PE1T; $\square$, phase transition; $\triangle$, melting of the high pressure phase. The hatched area means the high pressure phase of PE10T.

between PE10T and PE1T should decrease at elevated pressure because the molecular mobility of molten PE1T should decrease with pressure. The molecular motion of PE10T in the crystal should also decrease at elevated pressure. The hatched area in the phase diagram at $500 \mathrm{MPa}$ shows the temperature range of the high pressure phase of PE. The triple point of the high pressure phase in Figure 6 shifted to higher pressure with decreasing the fraction of PE10T. The marks $(\triangle)$ and $(x)$ in the phase diagram at $0.1 \mathrm{MPa}$ (Figure 7(a)) means the melting temperature of the PE10T sample crystallized at $500 \mathrm{MPa}$. It is considered that ECC is formed in PE10T even in the binary mixture with the weight fraction above 0.7 by 
Table II. Values of $A, B$, and $C$ in the quadratic equation, $T_{\mathrm{m}}=A+B P-C P^{2}$ for the melting temperature of PE10T and PE2T in the mixture of different weight fraction in run 1 of DTA up to $500 \mathrm{MPa}$

\begin{tabular}{|c|c|c|c|c|c|c|}
\hline \multirow{3}{*}{$W_{\mathrm{PE} 10 \mathrm{~T}}$} & \multicolumn{3}{|c|}{ PE10T } & \multicolumn{3}{|c|}{ PE2T } \\
\hline & $A$ & $B$ & $C$ & $A$ & $B$ & $C$ \\
\hline & ${ }^{\circ} \mathrm{C}$ & $\mathrm{K} \mathrm{MPa}^{-1}$ & $\mathrm{~K} \mathrm{MPa}^{-2}$ & ${ }^{\circ} \mathrm{C}$ & $\mathrm{K} \mathrm{MPa}^{-1}$ & $\mathrm{KMPa}^{-2}$ \\
\hline 1.0 & 131.0 & 0.302 & $1.729 \times 10^{-4}$ & & & \\
\hline 0.9 & 131.3 & 0.317 & $2.081 \times 10^{-4}$ & & & \\
\hline 0.8 & 130.6 & 0.307 & $1.913 \times 10^{-4}$ & 124.0 & 0.286 & $1.583 \times 10^{-4}$ \\
\hline 0.7 & 129.4 & 0.307 & $1.870 \times 10^{-4}$ & 125.7 & 0.294 & $1.762 \times 10^{-4}$ \\
\hline 0.6 & 129.9 & 0.291 & $1.465 \times 10^{-4}$ & 124.6 & 0.277 & $1.511 \times 10^{-4}$ \\
\hline 0.5 & 129.6 & 0.296 & $1.613 \times 10^{-4}$ & 125.2 & 0.283 & $1.544 \times 10^{-4}$ \\
\hline 0.4 & 129.8 & 0.298 & $1.623 \times 10^{-4}$ & 125.6 & 0.273 & $1.300 \times 10^{-4}$ \\
\hline 0.3 & 128.3 & 0.300 & $1.740 \times 10^{-4}$ & 125.1 & 0.291 & $1.679 \times 10^{-4}$ \\
\hline 0.2 & 126.1 & 0.310 & $1.939 \times 10^{-4}$ & 124.2 & 0.304 & $1.908 \times 10^{-4}$ \\
\hline 0.1 & 126.0 & 0.300 & $1.730 \times 10^{-4}$ & 124.9 & 0.286 & $1.576 \times 10^{-4}$ \\
\hline 0.0 & & & & 125.5 & 0.295 & $1.792 \times 10^{-4}$ \\
\hline
\end{tabular}

the crystallization from the melt at $500 \mathrm{MPa}$. Therefore, the phase diagram of melting of the binary mixture changes with the crystallization condition.

Pressure change of the DTA melting curve of pure PE2T was also studied at pressures up to $500 \mathrm{MPa}$. Single endothermic peak of melting was observed up to $500 \mathrm{MPa}$ and the peak only shifted to higher temperature with pressure as observed in pure PE1T. The peak intensity and peak shape did not change with pressure up to $500 \mathrm{MPa}$ and no phase transition occurred in the sample of PE2T. It is recognized that the high pressure phase of PE begins to appear above the molecular weight 2000 . The carbon number of PE of the molecular weight 2000 is about 142 . This value of carbon number is very close to the limit of the occurrence of chain folding in $\mathrm{PE}^{12}$ but it is considered that PE2T formed from the melt at $0.1 \mathrm{MPa}$ is extended chain crystal.

Pressure change of the DTA melting curve of the binary mixture of PE10T and PE2T was also studied and the pressure dependence of $T_{\mathrm{m}}$ of PE10T and PE2T in the mixture was determined. Pressure dependence of $T_{\mathrm{m}}$ of PE10T and PE2T also fits the quadratic equation. The values of $A, B$, and $C$ determined by the least squares method were listed in Table II. The value of $A$ for PE10T in this binary mixture is larger than the value for the binary mixture of PE10T/PE1T with the weight fraction below $W(\mathrm{PE} 10 \mathrm{~T})=0.6$. The lower molecular weight PE1T is more effective in decreasing $T_{\mathrm{m}}$ of PE10T compared with PE2T. The value of $B$ for PE10T in this mixture is almost the same as that of the mixture of $\mathrm{PE} 10 \mathrm{~T} / \mathrm{PE} 1 \mathrm{~T}$. The value of $B$ for PE2T in the mixture is slightly larger than that of PE1T in the mixture of PE10T/PE1T.

The phase diagrams of the binary mixture of PE10T/PE2T at elevated pressure are obtained by the same method described in PE10T/PE1T binary mixture. The phase diagram at 0.1 and $500 \mathrm{MPa}$ is shown in Figures 8 (a) and (b). At $0.1 \mathrm{MPa}, T_{\mathrm{m}}$ of PE10T decreased with decreasing the weight fraction below 0.5 but the depression rate is small compared with PE10T/PE1T mixture. $T_{\mathrm{m}}$ of PE2T did not change with the weight fraction. The temperature difference between $T_{\mathrm{m}}$ of PE10T and PE2T increased slightly at $500 \mathrm{MPa}$. Taking into account that two peaks still exist in the sample of $W(\mathrm{PE} 10 \mathrm{~T})=0.1$ at $500 \mathrm{MPa}$ and the difference of $T_{\mathrm{m}}$ increased with pressure, this binary mixture is not 
(a)

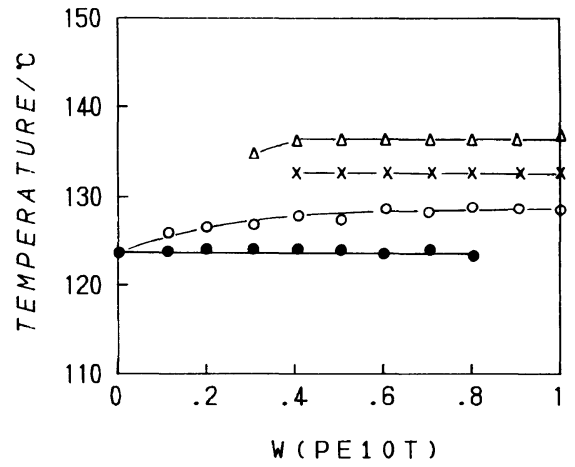

(b)

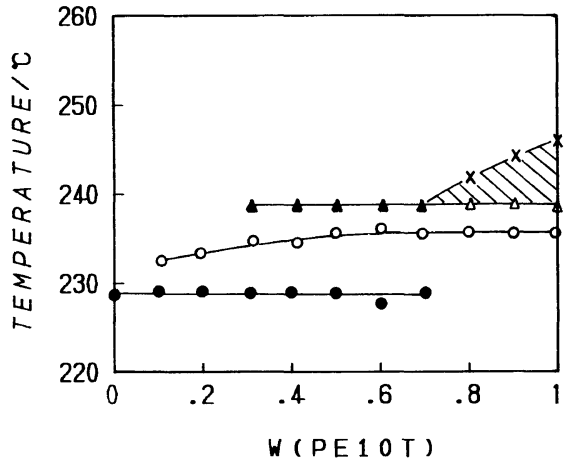

Figure 8. (a): Phase diagram of melting of the binary mixture of PE10T and PE2T at $0.1 \mathrm{MPa}$. O, PE10T crystallized at $0.1 \mathrm{MPa}$; $\mathrm{PE} 2 \mathrm{~T} ; \times, \triangle$, PE10T crystallized at $500 \mathrm{MPa}$.

(b): Phase diagram of melting of the binary mixture of PE10T and PE2T at $500 \mathrm{MPa}$. O, PE10T crystallized at $0.1 \mathrm{MPa}$;, PE2T; $\triangle$, phase transition; $\times$, melting of the high pressure phase; $\boldsymbol{\Delta}$, melting of ECC.

completely eutectic system. In completely eutectic system of $\mathrm{C} 30$ and $\mathrm{C} 22$ binary mixture, the eutectic point still exists at $500 \mathrm{MPa}$ in the phase diagram. ${ }^{10}$ The hatched area in the phase diagram at $500 \mathrm{MPa}$ shows also the high pressure phase of PE10T. The high melting temperature peak corresponding to the melting of ECC $(\boldsymbol{A})$ is observed in the range between 0.7 and 0.3 in $W(\mathrm{PE} 10 \mathrm{~T})$. The melting peak also appeared in the phase diagram at $0.1 \mathrm{MPa}$ of the binary mixture crystallized at $500 \mathrm{MPa}(\triangle$ in Figure 8(b)).

In the binary mixture of PE2T and PE1T, two endothermic peaks due to the melting of PE2T and PE1T appeared in the DTA curve up to $500 \mathrm{MPa}$. Pressure dependence of $T_{\mathrm{m}}$ of PE2T and PE1T in the mixture was also determined. The values of $A, B$, and $C$ in the quadratic equation are listed in Table III. $T_{\mathrm{m}}$ of PE2T decreased from $125.5^{\circ} \mathrm{C}$ at $W(\mathrm{PE} 2 \mathrm{~T})=$ 1.0 to $117.1^{\circ} \mathrm{C}$ at $W(\mathrm{PE} 2 \mathrm{~T})=0.2$. Figures $9(\mathrm{a})$ and (b) show the phase diagram of PE2T/PE1T binary mixture at 0.1 and $500 \mathrm{MPa}$. In Figure $9(\mathrm{a})$, the eutectic point is close to $W(\mathrm{PE} 2 \mathrm{~T})=$ 0.1 in the weight fraction. The peak of the DTA curve of melting in the binary mixture of $W(\mathrm{PE} 2 \mathrm{~T})=0.1$ at $0.1 \mathrm{MPa}$ is broader than the peak of melting of pure PE2T, so that the peak of melting of the mixture of PE2T/PE1T may be composed of eutectic melting and the melting of residual PE2T crystal. Smith and Manley $^{12}$ and Shu et al. ${ }^{13}$ reported that solid solution is formed in PE2T and PE1T binary mixture quenched in the dry ice/acetone but the eutectic phase diagram is observed if binary mixture is cooled down from the melt at the rate of $10^{\circ} \mathrm{C} \mathrm{min}^{-1}$. They predicted an eutectic point at 0.02 in volume fraction of PE2T using the Flory-Huggins theory. ${ }^{14}$ The eutectic point in this study is close to their data.

$T_{\mathrm{m}}$ of PE2T in the mixture decreased with decreasing the PE2T content due to the solvent effect of PE1T. The temperature difference between the $T_{\mathrm{m}}$ curve of PE2T and the eutectic temperature increased in the phase diagram at $500 \mathrm{MPa}$. It is considered that the solvent effect may decrease at elevated pressure due to the decreased mobility of molten PE1T molecule. This interpretation on the pressure change of the phase diagram of PE2T/PE1T binary mixture should be extended to the mixture of PE10T/PE1T and PE10T/PE2T.

\section{Effects of Mixing Low Molecular Weight PE on the High Pressure Phase Transition of PE10T}

The DTA curve of melting of PE under high pressure above $350 \mathrm{MPa}$ becomes complicated because the phase transition from orthorhombic phase to the hexagonal phase occurs below its melting temperature. ${ }^{11}$ In pure PE10T, three 
Table III. Values of $A, B$, and $C$ in the quadratic equation, $T_{\mathrm{m}}=A+B P-C P^{2}$ for the melting temperature of PE2T and PE1T in the mixture with different weight fraction in run 1 of DTA up to $500 \mathrm{MPa}$

\begin{tabular}{|c|c|c|c|c|c|c|}
\hline \multirow{3}{*}{$W_{\text {PE2T }}$} & \multicolumn{3}{|c|}{ PE2T } & \multicolumn{3}{|c|}{ PE1T } \\
\hline & $A$ & $B$ & $C$ & $A$ & $B$ & $C$ \\
\hline & ${ }^{\circ} \mathrm{C}$ & $\mathrm{KMPa}^{-1}$ & $\mathrm{KMPa}^{-2}$ & ${ }^{\circ} \mathrm{C}$ & $\mathrm{KMPa}^{-1}$ & $\mathrm{KMPa}^{-2}$ \\
\hline 1.0 & 125.5 & 0.295 & $1.792 \times 10^{-4}$ & & & \\
\hline 0.9 & 125.2 & 0.294 & $1.734 \times 10^{-4}$ & & & \\
\hline 0.8 & 125.1 & 0.289 & $1.746 \times 10^{-4}$ & & & \\
\hline 0.7 & 124.6 & 0.301 & $1.871 \times 10^{-4}$ & 110.5 & 0.288 & $1.716 \times 10^{-4}$ \\
\hline 0.6 & 124.0 & 0.299 & $1.784 \times 10^{-4}$ & 109.9 & 0.293 & $1.751 \times 10^{-4}$ \\
\hline 0.5 & 122.7 & 0.292 & $1.686 \times 10^{-4}$ & 109.1 & 0.290 & $1.779 \times 10^{-4}$ \\
\hline 0.4 & 120.8 & 0.278 & $1.556 \times 10^{-4}$ & 109.6 & 0.279 & $1.766 \times 10^{-4}$ \\
\hline 0.3 & 120.4 & 0.295 & $1.826 \times 10^{-4}$ & 108.9 & 0.297 & $1.974 \times 10^{-4}$ \\
\hline 0.2 & 117.1 & 0.270 & $1.374 \times 10^{-4}$ & 110.4 & 0.262 & $1.400 \times 10^{-4}$ \\
\hline 0.1 & & & & 109.8 & 0.260 & $1.312 \times 10^{-4}$ \\
\hline 0.0 & & & & 111.4 & 0.280 & $1.720 \times 10^{-4}$ \\
\hline
\end{tabular}

(a)

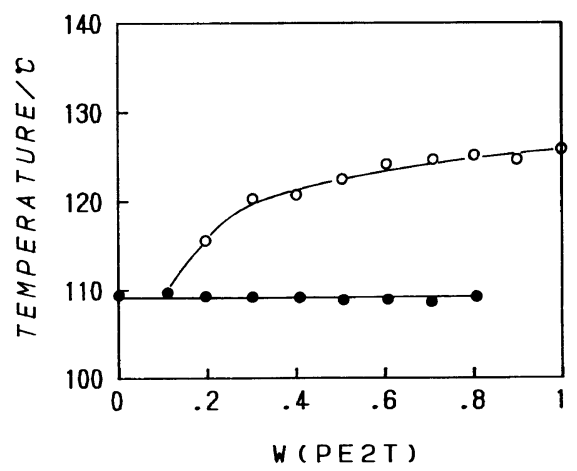

(b)

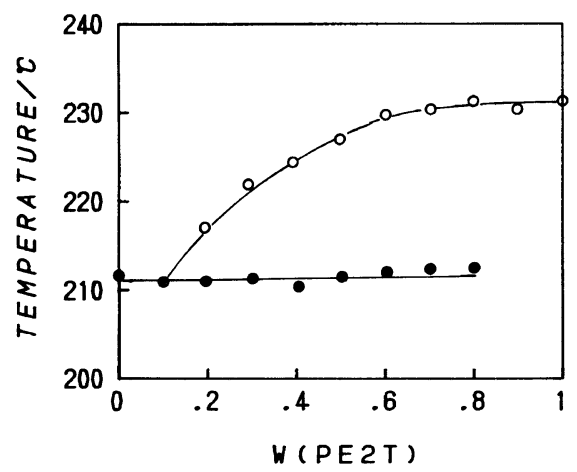

Figure 9. (a): Phase diagram of melting of the binary mixture of PE2T and PE1T at $0.1 \mathrm{MPa}$. O, PE2T; PE1T.

(b): Phase diagram of melting of the binary mixture of PE2T and PE1T at $500 \mathrm{MPa}$. O, PE2T; O, PE1T. peaks appeared in the DTA curve at $500 \mathrm{MPa}$ as shown in Figure 10. The origin of these peaks has been already described. In the DTA curve of melting of PE10T in PE10T/PE1T mixture of $W(\mathrm{PE} 10 \mathrm{~T})=0.8$, three peaks also appear around the melting temperature of PE10T. The peak at $240.6^{\circ} \mathrm{C}$ is due to the phase transition and the peak at $243.6^{\circ} \mathrm{C}$ is due to the melting of the high pressure phase. In the DTA curve of melting for $W(\mathrm{PE} 10 \mathrm{~T})=0.6$, a small peak appears at $241.8^{\circ} \mathrm{C}$. The peak at $233.9^{\circ} \mathrm{C}$ corresponds to the melting of $\mathrm{FCC}$ at $500 \mathrm{MPa}$ as shown in Figure 6, so that the high pressure phase of PE does not exist in the mixture of this weight fraction. In the case of $W(\mathrm{PE} 10 \mathrm{~T})=$ 0.7 , a peak due to the phase transition was observed, therefore the high pressure phase of $\mathrm{PE}$ is absent at $W(\mathrm{PE} 10 \mathrm{~T})=0.6$ at $500 \mathrm{MPa}$. In the sample of $W(\mathrm{PE} 10 \mathrm{~T})=0.5$, only the low temperature peak due to the melting of FCC of PE10T appeared. These experimental facts indicate that the orthorhombic PE10T in the mixture with PE1T melts directly below $W(\mathrm{PE} 10 \mathrm{~T})=0.6$ in the heating process. The same phenomena (disappearance of the high pressure phase of PE) were observed in PE of the molecular weight 67000 and high melting temperature diluent such as 1,2,4,5-tetra- 


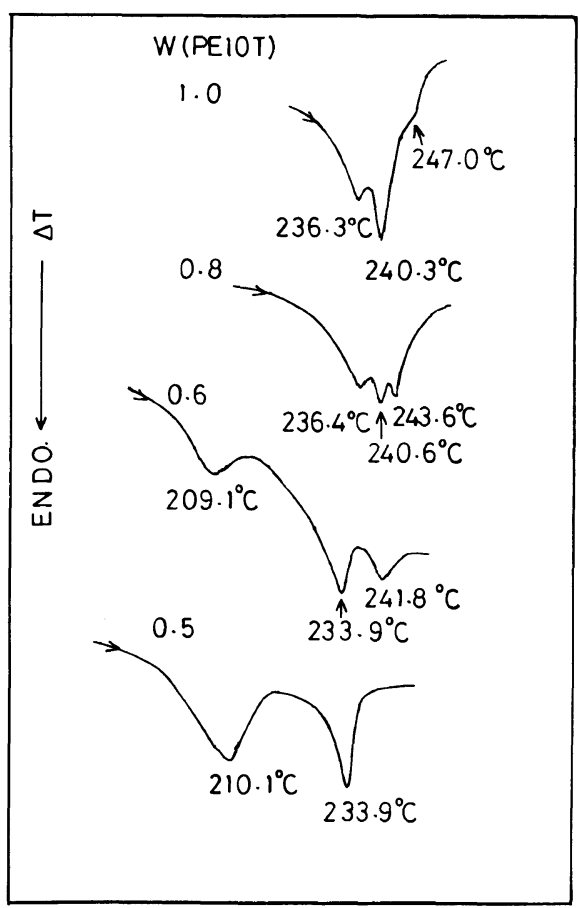

Figure 10. DTA curve of melting at $500 \mathrm{MPa}$ of pure PE10T and PE10T in the binary mixture with PE1T for different weight fractions.

chlorobenzene $^{9}$ or 1,3,5-tribromobenzene $\mathrm{e}^{10}$ for $W(\mathrm{PE})=0.8$. In the mixture with $\mathrm{C} 40$, the high pressure phase of $\mathrm{PE}$ exists to a slightly lower weight fraction. As the mechanism of the high pressure phase transition of PE, the role of the cross-linkages induced at the crossing points between $\mathrm{PE}$ chains by the reduction of free volumes under high pressure was proposed. These cross-linkages exist even at the high temperature region above true melting temperature of the orthorhombic phase and the hexagonal phase appeared. ${ }^{16}$ These considerations were also proposed by van Aerle and Lemstra. ${ }^{17}$ In the binary mixture of PE10T with PE1T or PE2T, PE1T and PE2T act as lubricant and impede the formation of entanglements or cross-linkages of PE molecules. It is clear that the high pressure phase of PE does not exist below $W(\mathrm{PE} 10 \mathrm{~T})=0.7$ in PE10T/PE1T mixture.

The formation of ECC of PE10T during cooling from the melt under high pressure is also affected by mixing PE1T and PE2T. In the DSC curve of melting of the high pressure crystallized PE10T sample, endothermic peak due to the melting of ECC appears at about $137.4^{\circ} \mathrm{C}$ as shown in Figure 4 . However, the peak due to the melting of ECC disappears below the weight fraction of $\mathrm{PE} 10 \mathrm{~T}=0.5$ in $\mathrm{PE} 10 \mathrm{~T} / \mathrm{PE} 1 \mathrm{~T}$ mixture. It is considered that ECC of PE is formed under a condition of decreased molecular mobility but PE1T and PE2T act as lubricant and the mobility of PE molecule again increased. The crystallization temperature of PE1T and PE2T is lower than that of PE10T, so that PE10T crystallizes in the molten low molecular weight $P E$ even under high pressure. $T_{\mathrm{m}}$ of ECC of PE10T in the mixture is plotted in the phase diagram (Figures 7 and 9). It is clearly recognized that lower molecular weight PE1T interferes the formation of ECC of PE10T stronger than PE2T.

\section{CONCLUSIONS}

1. Pressure change of the phase diagram of melting of the three binary mixtures of low molecular weight $\mathrm{PE}$ was determined at pressures up to $500 \mathrm{MPa}$. The Phase diagram of the binary mixture of PE10T/PE1T and PE10T/PE2T is not eutectic and only $T_{\mathrm{m}}$ depression of PE10T is observed. $T_{\mathrm{m}}$ of the low molecular weight $\mathrm{PE}$ did not change with the change of the weight fraction at $0.1 \mathrm{MPa}$. The region between $T_{\mathrm{m}}$ depression curve of PE10T and $T_{\mathrm{m}}$ line of PE1T and PE2T increased with increasing pressure, suggesting the decrease in the molecular mobility of the low molecular weight (lower $T_{\mathrm{m}}$ ) component.

The phase diagram of PE2T/PE1T binary mixture is eutectic type and the eutectic point is very close to 0.1 in $W(\mathrm{PE} 2 \mathrm{~T})$. The phase diagram changed slightly with pressure because the pressure dependence of $T_{\mathrm{m}}$ of PE2T and PE1T is very close. The eutectic point did not change with pressure.

2. The high pressure phase transition oc- 
curs in pure PE10T but it does not occur in the lower weight fraction of PE10T in the mixture with PE2T and PE1T. The high pressure phase region of PE10T decreased with increasing PE1T or PE2T in the binary mixture. Mixing PE1T is more effective for the disappearance of the high pressure phase of PE10T than PE2T. At $500 \mathrm{MPa}$, the high pressure phase of PE10T is not found below $W(\mathrm{PE} 10 \mathrm{~T})=0.6$.

3. ECC of pure PE10T is formed by crystallization from the melt at above $400 \mathrm{MPa}$. However, the formation of ECC of PE10T is impeded by mixing low molecular weight PE. In the mixture of PE10T/PE1T, ECC of PE10T was not formed below $W(\mathrm{PE} 10 \mathrm{~T})=0.5$. The role of the molten low molecular weight $\mathrm{PE}$ is considered as lubricant.

\section{REFERENCES}

1. H. A. Nechitailo, A. B. Tonchev, P. M. Rozenberg, and E. M. Terentueba, J. Phys. Chem. USSR (in Russian), 24, 2694 (1960).

2. G. I. Asbach, H.-G. Kilian, and Fr. Stracke, Colloid
Polym. Sci., 260, 151 (1982).

3. H.-G. Kilian, Makromol. Chem., 116, 2194 (1968).

4. I. Denicolo, A. F. Craievich, and J. Doucet, J. Chem. Phys., 82, 6200 (1984).

5. D. L. Dorcet, Macromolecules, 19, 2965 (1986).

6. W. P. Zhang and D. L. Dorcet, J. Polym. Sci., B, Polym. Phys., 28, 1223 (1990)

7. B. Wunderlich, "Macromolecular Physics," Vol. 3, Academic Press, New York, N.Y., 1980.

8. A. Nakajima and F. Hamada, Kolloid Z. Z. Polym., 205, 55 (1965).

9. S. J. Organ and A. Keller, J. Polym. Sci., B, Polym. Phys., 24, 2319 (1986).

10. C. Nakafuku and T. Sugiuchi, Polymer, 34, 4945 (1993).

11. C. Nakafuku, M. Yasuniwa, and S. Tsubakihara, Polym. J., 22, 110 (1990).

12. Y. Urabe, T. Iida, and H. Yamamoto, Polym. Prepr. Jpn. (in Japanese), 43, 3301 (1994).

13. P. Smith and R. St. J. Manley, Macromolecules, 12, 483 (1979).

14. P. H. C. Shu, D. J. Burchell, and S. L. Hsu, J. Polym. Sci., Polym. Phys. Ed., 18, 1421 (1980).

15. P. J. Flory, "Principles of Polymer Chemistry," Cornell University Press, Ithaca, N.Y., 1953, 568.

16. K. Nagata, K. Tagashira, S. Taki, and T. Takemura, Jpn. J. Appl. Phys., 19, 985 (1980).

17. N. A. J. M. van Aerle and P. J. Lemstra, Polym. J., 20, 131 (1988). 